\title{
Educación y Sostenibilidad en la Universidad de Valencia: construyendo futuro desde el pasado
}

\author{
Pilar Aznar Minguet \\ Universitat de Valencia. España.pilar.aznar@uv.es \\ ORCID: https://orcid.org/0000-0001-7851-3808 \\ M. Ángeles Ull Solís \\ Universitat de Valencia. España. angels.ull@uv.es \\ ORCID:https://orcid.org/0000-0003-0244-3596
}

[Recibido: 7 Diciembre 2018. Revisado: 11 Enero 2019. Aceptado: 17 Enero 2019]

\begin{abstract}
Resumen: Una práctica académica de calidad en educación superior requiere estructuras institucionales que apoyen los principios de la Sostenibilidad y los lleven a la práctica, pero también requiere profesorado que esté motivado y capaz de impregnar su forma de enseñar y las materias que enseña desde criterios y valores relacionados con la sostenibilidad. El contexto de este trabajo es la educación e investigación para la sostenibilidad (EIpS) en la Universitat de València (UV) en los últimos 12 años. El método utilizado ha sido el Análisis de documentos que siguiendo los criterios de pertinencia, exhaustividad y actualidad ha permitido encontrar la información necesaria para desarrollar el estudio planteado. La finalidad se dirige a construir un relato de la trayectoria que ha seguido la EIpS en la UV, destacando sus avances, retrocesos, y las causas y consecuencias de los mismos, para poder plantear una perspectiva de futuro desde la óptica de los ODS. Como conclusiones cabe señalar: a) que la sostenibilización requiere decisiones políticas que permitan generar espacios para la participación de los diversos estamentos universitarios en la definición de estrategias institucionales que respondan a los objetivos y valores relacionados con la sostenibilidad; y b) se plantea que la aprobación de los ODS 2030, desde una mirada crítica, es una nueva palanca para seguir adelante en el camino emprendido hace más de 20 años, porque concreta en los 17 ODS formas de trabajar todos los aspectos de la sostenibilidad que permiten superar algunos de los obstáculos para el avance hacia un futuro más sostenible.
\end{abstract}

Palabras clave: Educación para la Sostenibilidad, Sostenibilidad y Educación superior. Formación de formadores.

\section{Education and Sustainability at the University of Valencia: Building future from the past}

Abstract: Higher education requires institutional structures that support the principles of Sustainability and put them into practice. It is also necessary a faculty that is motivated and able to permeate the way they teach and the subjects they teach from criteria and values related to sustainability. The context of this work is education and research for sustainability (ERfS) at the University of Valencia (UV) in the last 12 years. The method used has been the Analysis of documents with criteria of relevance, completeness and topicality, has allowed to find the necessary information to develop the proposed study. The purpose is to build a story of the trajectory that the ERfS has followed in the UV to be able to propose a future perspective from the perspective of the SDGs. The conclusions are: a) that sustainability requires political decisions that allow the participation of the different university levels in the definition of institutional strategies that respond to the objectives and values related to sustainability and b) The approval of SDGs 2030, from a critical perspective, is a new lever to move forward on the road undertaken more than 20 years ago, because it specifies in the 17 SDGs ways to work on all aspects of sustainability that allow us to overcome some of the obstacles to progress towards a more sustainable future.

Keywords: Sustainability Education, Sustainability and Higher Education, Training of trainers 
Para citar este artículo: Aznar Minguet, P. y Ull Solís, A. (2019). Educación y Sostenibilidad en la Universidad de Valencia: construyendo futuro desde el pasado. Revista de Educación Ambiental y Sostenibilidad 1(1), 1202. doi: 10.25267/Rev_educ_ambient_sostenibilidad.2019.v1.i1.1202

\section{Introducción}

Existen varias referencias a nivel nacional e internacional de la coordinación de las universidades interesadas en la introducción de la Sostenibilidad en la docencia, la investigación, la gestión y las relaciones con la sociedad que las rodea, que se han concretado en diversas declaraciones internacionales que ya se han citado ampliamente en otros artículos (Ull, Martínez, Piñero y Aznar, 2010; Junyent, Bonil y Calafell, 2011; Lozano, Merril, Sammalisto, Ceulemans y Lozano, 2017). Desde esta óptica tratamos de analizar, en este artículo, la trayectoria de la Educación para la sostenibilidad en la UV, para conocer la tendencia seguida y poder plantear líneas de actuación futuras.

Pero el progreso en la reorientación del currículum hacia la Sostenibilidad ha sido lento (Tilbury, 2012a, 2012b). Y la literatura y la investigación en esta área muestra aún pocos ejemplos exitosos en lo referente a cambios a gran escala (De Harpe y Thomas, 2009). La Universitat de València (UV) cuenta actualmente con unos 50000 alumnos (40000 de grado y 10000 de postgrado y de ellos 6000 de máster y 4000 de doctorado), más de 4200 profesores y más de 1900 personas de administración y servicios. Está constituida por 18 centros (17 Facultades y una Escuela de Ingenierías), distribuidos en tres campus universitarios; se imparten actualmente un total de 53 titulaciones de grado y 118 másters oficiales de postgrado y 75 programas de doctorado.

La UV ya en los Estatutos de 1986 y en los actuales aprobados en 2003, recoge entre sus objetivos "que está al servicio de la paz, de la igualdad entre las mujeres y los hombres y la defensa ecológica del medio" y ha ido implantando estudios de grado, postgrado y másters relacionados con estos temas de forma paulatina.

En 1996 se constituyó por parte del rectorado y a instancias de diversas asociaciones de estudiantes, una Delegación del rector para temas de Medio Ambiente, con un primer plan de acción que contempló:

a) Acciones de gestión, plasmadas, entre otras, en el Programa de Minimización de Residuos.

b) Acciones para favorecer la investigación y actualización profesional: por ejemplo subvención de trabajos de investigación sobre la propia universidad (desde cómo implementar un plan de energía solar, que fue el embrión del actual parque fotovoltaico de los techos de los edificios, funcionando desde 2008, hasta la inclusión de la sostenibilidad en el currículo, entonces denominada ambientalización curricular, que se inició en los estudios de Pedagogía.

c) Acciones de participación y cooperación, con la puesta en marcha de equipos de voluntariado universitario en educación ambiental con participación activa en el plan de Minimización de Residuos y otros temas (senderos de vegetación, participación en la vigilancia para la prevención de incendios forestales en verano ...) 
d) Acciones de formación, con la puesta en marcha del Programa Ambientaliza tu curriculum.

A partir del año 2002, la Delegación se integró en el Servicio de Seguridad, Salud y Calidad Ambiental; se creó una plaza de técnico de gestión ambiental y se prosiguió con las acciones iniciadas en 1996. Tal y como indicaba Capdevila (1999), en su temprano análisis basado en referentes internacionales de la ambientalización de las universidades españolas, la ambientalización es un compromiso colectivo que no puede depender de una o dos personas y ya entonces ponía ejemplos de cómo los programas pueden quedar marginados con los cambios en los equipos de dirección.

En 2010, hubo un cambio sustancial con la elección de un nuevo equipo rectoral que supuso la constitución de un vicerrectorado específico dedicado a la Sostenibilidad, lo que indica la importancia que empieza a adquirir el tema, confluyendo con lo que está ocurriendo también en muchas universidades españolas y del resto del mundo. En las cuales se asume que para garantizar una práctica académica de calidad en educación superior se requieren estructuras institucionales que apoyen los principios de la Sostenibilidad y los lleven a la práctica, pero también se requiere profesorado que esté motivado y capaz de impregnar su forma de enseñar y las materias que enseñan desde criterios y valores relacionados con la sostenibilidad (UNECE, 2013; Barth, 2014).

\section{Contexto del estudio: El Proyecto Campus Sostenible/UV}

En Julio de 2010, a iniciativa del recién creado Vicerrectorado de Sostenibilidad, un grupo de 30 expertos de la comunidad universitaria comenzó un proceso de análisis y diagnóstico de la situación y necesidades de la UV en materia de Sostenibilidad con el fin de elaborar una estrategia institucional para la gestión coordinada de las actividades universitarias desde criterios sostenibles. La metodología utilizada conformó una estructura descriptivo-exploratoria y evaluativa basada en un análisis DAFO, centrado, por una parte, en las debilidades y fortalezas que pueden presentar con carácter propio la aplicación de iniciativas relacionadas con la sostenibilidad y por otra en el marco de oportunidades y amenazas a las que enfrentarse. Esta iniciativa supuso un punto de inflexión en el desarrollo de la sostenibilidad en la UV. El proceso del análisis se focalizó, de manera consensuada por los expertos, en 12 temas clave en materia de sostenibilidad:

1. Educación y sensibilización para la sostenibilidad; 2. Investigación para la sostenibilidad; 3. Accesibilidad universal; 4. Edificación, confort y ergonomía; 5. Eficiencia energética y gestión de los consumos; 6. Gestión de los residuos, vertidos y emisiones; 7. Planeamiento urbanístico y Campus (jardines y espacios comunes) y ruido ambiental; 8. Criterios de sostenibilidad en las prescripciones y en los criterios de adjudicación de todo tipo de contratos de bienes y servicios; 9. Promoción de la Salud; 10. Voluntariado y participación; 11. Movilidad sostenible y 12. Comercio justo.

El objetivo de esta revisión era conseguir que la Sostenibilidad fuera reconocida como una dimensión transversal que formara parte de la perspectiva desde la que abordar la mayoría de las actuaciones universitarias y, muy especialmente, del modelo social de campus que se quería desarrollar. Para ello los diferentes grupos de trabajo se reunieron a lo largo de un año en 20 sesiones para elaborar un plan, que salió a información pública de toda la comunidad universitaria; fue por tanto un proceso 
participativo en el que se intentó recoger todas las aportaciones realizadas, así como las políticas que ya se estaban desarrollando en estos ámbitos. Los datos obtenidos permitieron describir la situación inicial de partida respecto a la innovación y mejora institucional de las actividades universitarias en materia de Sostenibilidad y facilitar la puesta en marcha del compromiso institucional, reflejado en el Proyecto Campus Sostenible / UV 2015 (2011) con el objetivo de contribuir a la Sostenibilidad desde todos los ámbitos que caracterizan a la institución: formación, investigación, gestión propia y relaciones con la sociedad. Aprobado finalmente en el Consejo de Gobierno de la universidad, era un programa de acción con horizonte 2015. Sus objetivos han sido:

- Sensibilizar y formar a la comunidad universitaria en materia de Sostenibilidad.

- Evaluar las competencias específicas relacionadas con la Sostenibilidad en los programas de formación y hacer un seguimiento de su implantación.

- Conseguir una administración universitaria y un campus más sostenible y saludable, optimizando los recursos disponibles, estableciendo metas cuantitativas plurianuales y evaluando el impacto de sus actividades e instalaciones.

- Contribuir, en los ámbitos de actuación que le corresponden al desarrollo sostenible de la sociedad.

La Universitat de València, a través de este plan se ha dirigido a optimizar sus recursos para contribuir, en el marco de los planes operativos anuales, a las estrategias europea y española de desarrollo sostenible. Estas estrategias han planteado cinco dimensiones de actuación: cultural, ambiental, económica, institucional y social, que han dado cobertura en la práctica a todas las actividades en materia de Sostenibilidad de la organización. Estas dimensiones se han concretado en 25 programas de actuación. La dimensión cultural incluye la Educación e investigación para la salud y la sostenibilidad y se estructura en 4 programas.

Desde las dimensiones ambiental, económica e institucional, reunidas bajo el epígrafe de Campus Sostenible y Saludable, la Universidad se ha dirigido a minimizar el impacto negativo sobre el medio ambiente, al mismo tiempo que maximiza la creación de valor económico, social y ambiental y satisfacer los requerimientos de la comunidad universitaria para poder alcanzar un campus sostenible y saludable. Incluye 17 programas de actuación.

Por último, la dimensión social, Compromiso social y participación, dirigida al impacto social de la Universidad y de sus actividades tanto a nivel interno como externo, adoptando medidas de compromiso social y participación. Contempla 4 programas de actuación.

Se constituyó una Comisión de Sostenibilidad con las funciones de asesorar a los órganos de gobierno en esta materia, impulsar las actuaciones del proyecto Campus Sostenible, coordinar la política de información y comunicación, evaluar y hacer un seguimiento de los programas y crear e impulsar la actividad de un observatorio de sostenibilidad. Este proyecto se desarrolló durante los años 2012 a 2015 impulsado por el Vicerrectorado de Sostenibilidad e Infraestructuras. En 2016, las competencias en Sostenibilidad pasaron al Vicerrectorado de Ordenación Académica, Profesorado y 
Sostenibilidad, quedando diluidas por un tiempo, hasta qué con el cambio de rectorado en 2017, se ha creado un Vicerrectorado de Igualdad, Diversidad y Sostenibilidad desde el que se están retomando acciones de cara a reimpulsar nuevas iniciativas relacionadas con la sostenibilidad. Este es el contexto del trabajo que presentamos en este artículo y desde el cual nos centramos en la primera dimensión: Educación e investigación para la Sostenibilidad.

\section{Metodología y objetivos específicos del estudio}

El método utilizado ha sido el Análisis de documentos como técnica de investigación que nos permite encontrar la información necesaria para desarrollar el estudio planteado, mediante un registro estructurado de la información contenida en los documentos analizados. Esta técnica conlleva tres procesos: a) de comunicación, que permite recuperar información para transmitirla, b) de transformación mediante las operaciones de análisis de la información recopilada; y c) un proceso analíticosintético que permite estudiar, interpretar y sintetizar la información de acuerdo con los objetivos planteados en el estudio.

El análisis de los documentos, que ha seguido los criterios de pertinencia, exhaustividad y actualidad, se ha centrado en cuatro tipos de documentos: a) Documentos internos del vicerrectorado, con competencias en sostenibilidad, promotor del Proyecto Campus Sostenible: Actas de la Comisión de Sostenibilidad, solicitudes de aprobación de acciones relacionadas con la sostenibilidad dirigidas al Consejo de Gobierno de la Universidad, documentos relacionados con la participación de la UV en la Comisión de Sostenibilidad de la CRUE; b) Información pública contenida en el apartado Sostenibilidad de la web de la UV: Informes sobre la inclusión de la sostenibilidad en los planes de estudio, informes sobre las acciones relacionadas con la sostenibilidad propuestas y realizadas; c) Información sobre los planes de estudio y guías docentes contenidas en la web de la UV; y d) Publicaciones del profesorado de la UV sobre temas relacionados con educación y sostenibilidad que se centren en la propia UV.

La finalidad del presente estudio se dirige a construir un relato de la trayectoria que ha seguido la educación y la investigación para la sostenibilidad en la UV, destacando sus avances, retrocesos, y las causas y consecuencias de los mismos. Y los objetivos específicos van dirigidos a:

a) Poner en valor la evolución de la introducción de la educación para la sostenibilidad en la UV en la docencia y la investigación, potencialmente transferible a otras universidades.

b) Revisar propuestas de acciones relacionadas con la sostenibilidad no realizadas.

c) Plantear una perspectiva de futuro desde la óptica de los ODS.

\section{Resultados: Desarrollo de la investigación y la educación para la sostenibilidad en la UV}

Este desarrollo viene precedido por una serie de estudios previos:

1. Diagnóstico de la situación inicial sobre la introducción de la sostenibilidad en la docencia. Se pretendía conocer la predisposición del profesorado ante lo que en un 
primer momento se denominó ambientalización curricular y el grado de conocimientos y formación en el tema. En este sentido se realizó un análisis previo de las preconcepciones del profesorado universitario respecto a la introducción de la variable "sostenibilidad" en la docencia e investigación, y un estudio de la situación inicial de los programas de las asignaturas en relación a la sostenibilidad, de los diferentes planes de estudio de tres centros piloto, uno de cada uno de los tres campus; la Facultad de Química en el Campus de Burjassot (área CientíficoTecnológica), la Facultad de Sociología en al campus del área de Ciencias Sociales y la Facultad de Geografía, en al campus del área de Humanidades. Este estudio permitió conocer el grado de ambientalización curricular de las licenciaturas seleccionadas como piloto, para diagnosticar la situación de partida.

Los resultados de este estudio se han recogido en varias publicaciones: Palacios y Aznar Miguet 2004; Piñero, Ull, Aznar y Martínez, 2006; Ull, Aznar, Martínez Agut, Palacios y Piñero, 2009; Ull, Martínez Agut, Piñero y Aznar Minguet, 2010; Aznar Minguet, Martínez-Agut, Palacios, Piñero y Ull, 2011.

2. Realización de seminarios de Diálogo disciplinar con el objetivo de introducir competencias para la sostenibilidad en los curricula. Para implicar en este proceso al profesorado, se solicitó la colaboración de los decanatos, facilitando la realización de una Jornada sobre sostenibilidad en la docencia que fue estimulada por la presentación de una ponencia sobre procesos similares realizados en otras universidades. A partir de ese seminario introductorio se invitó al profesorado interesado en constituir grupos de trabajo permanente junto con los miembros del equipo investigador para revisar sus programas a la luz de los temas propuestos por la UNESCO en el documento de la Década de la Educación para el Desarrollo Sostenible (DEDS), y definir las competencias generales de cada titulación relacionadas con la sostenibilidad. En todos los Grupos de discusión de las diversas titulaciones se trabajó la propuesta inicial de un modelo de competencias básicas o generales para la sostenibilidad, que ha sido recogida en esta universidad como referente y que los diversos grupos han discutido y adaptado a sus titulaciones. Este modelo concentra de forma interrelacionada 4 competencias básicas (Aznar Minguet, 2006): a) Comprensión crítica de la problemática ambiental global y local; b) Adquisición de habilidades, estrategias, técnicas y procedimientos para la toma de decisiones y la realización de acciones relacionadas con la sostenibilidad; c) Desarrollo de actitudes y valores relacionados con la sostenibilidad y d) Capacidad de relacionarse con el medio.

Fruto de estos trabajos son las publicaciones: Martínez, Aznar, Ull y Piñero, 2007; Piñero, Ull, Martínez y Aznar, 2007.

En el año 2008 se produjo un cambio en el marco de referencia de las titulaciones universitarias ya que, durante el mismo se diseñaron los nuevos planes de estudio de las titulaciones, que pasaron a denominarse Grados; dichos planes fueron aprobados por el Consejo de Gobierno de la universidad y verificados por la ANECA. Durante el período de información pública en la universidad se presentaron alegaciones para que se recogiera más ampliamente la sostenibilidad en estos títulos y se realizó también la revisión de los planes de grado de todas las titulaciones y, en su caso, las alegaciones pertinentes respecto a la introducción de competencias para la 
sostenibilidad, tomando como base el R.D. 1393/2007, de 29 de octubre, por el que se establece la ordenación de las enseñanzas universitarias oficiales.

Desde este nuevo marco de referencia, cabe citar la contribución al desarrollo de la investigación y educación para la sostenibilidad desde el estudio realizado sobre la Introducción de competencias para la sostenibilidad en los planes de estudio. Este estudio trata de especificar competencias básicas y adoptar criterios de sostenibilidad para reformular y orientar los programas curriculares en las titulaciones de Educación Infantil y Educación Primaria de la UV; se sitúa en el enfoque de la UNESCO de la educación para el desarrollo humano sostenible. La finalidad general es formar a estudiantes en competencias básicas para la sostenibilidad, para que una vez egresados, puedan tomar decisiones y realizar acciones profesionales (y personales) desde criterios de sostenibilidad. Y el objetivo es aportar la formación básica para el desarrollo de competencias para la sostenibilidad en los procesos de enseñanza/aprendizaje y la aplicación de criterios de sostenibilidad en la docencia. Esta cuestión ha sido tratada por diversos autores, que vienen realizando investigaciones sobre las competencias clave relacionadas con la Sostenibilidad en los estudios superiores (Sterling, 2005; Martínez-Agut et al., 2007; Junyent y De Ciurana, 2008; Lozano García, Gindara, Perni, Manzano y Hernández, 2008; Aznar Minguet y Ull, 2009; Sibbel, 2009; Thomas, 2009; Barrón, Navarrete y Ferrer-Balas, 2010; Aznar et al., 2011; Wiek, Withycombe y Redman, 2011; Barth y Rieckmann, 2012; Vilches y Gil, 2012; Ull, 2014).

Se aplicaron técnicas de obtención de datos mediante el uso de cuestionarios dirigidos a profesores y estudiantes, para conocer la realidad; así como técnicas de trabajo colaborativo y cooperativo y de grupos de Diálogo Disciplinar para elaborar estrategias específicas para la introducción de la sostenibilidad en la docencia.

Se realizó un Estudio sobre la ambientalización curricular en las titulaciones de Maestro de Educación Infantil y Maestro de Educación Primaria: preconcepciones y actitudes del profesorado. Y también un Estudio sobre las actitudes y comportamientos en relación a la sostenibilidad de los/as estudiantes de ambas titulaciones.

Como resultados, se identificaron los posibles problemas y cuestiones que en materia de sostenibilidad planteaba el profesorado en los ámbitos estudiados. Se determinaron los niveles de percepción del profesorado universitario respecto al desarrollo humano ambiental y socialmente sostenible. Se evaluó el nivel de conocimientos y actitudes del profesorado universitario respecto a la sostenibilización curricular. Y se detectaron las principales características del comportamiento del profesorado universitario ante la introducción de la sostenibilidad curricular en las titulaciones estudiadas.

En relación con los estudiantes: Se identificaron los posibles problemas y cuestiones que planteaban en materia de Sostenibilidad; se determinaron los niveles de percepción de los estudiantes respecto al desarrollo humano ambiental y socialmente sostenible; se evaluó el nivel de conocimientos y actitudes de los estudiantes respecto a la sostenibilización curricular; y se detectaron las principales características del comportamiento de los estudiantes ante los principales problemas socio-ambientales. 
Finalmente, para la evaluación se aplicó la técnica de entrevista en formato cuestionario abierto dirigido a los profesores participantes en las sesiones del Seminario de Diálogo Disciplinar.

Los resultados de este estudio están recogidos en diversas publicaciones: Aznar Minguet, Ull, Piñero y Martínez Agut, 2014a; 2014b; Martínez Agut, Ull y Aznar Minguet, 2014; Ull, Martínez Agut, Piñero y Aznar Minguet, 2014; Ull, Piñero, Martínez Agut y Aznar Minguet, 2014.

A partir de estos resultados, cabe citar aquí la realización de las siguientes acciones:

a) Oferta integrada de asignaturas sobre estudios de sostenibilidad desde el año 2012, con la finalidad de introducir los conocimientos básicos desde la perspectiva de la sostenibilidad, con carácter transversal, para la adecuada formación de los estudiantes. Esta oferta integrada de Estudios de Sostenibilidad incluye una materia con cuatro asignaturas, diseñadas y adaptadas a las áreas de conocimiento de las diferentes ramas del saber: Sostenibilidad, Ciencia y Tecnología; Sostenibilidad, Cultura y Educación; Sostenibilidad y Salud, y Sostenibilidad y Sociedad. Estas cuatro asignaturas comparten una troncalidad común, incluyendo, además, contenidos específicos coherentes con sus diferentes perspectivas disciplinares. Cada una de las cuatro asignaturas a impartir en las diferentes ramas consta de seis créditos, con reconocimiento académico por participación en actividades universitarias aprobado por el Consejo de Gobierno en 2010. Actualmente se siguen impartiendo la asignatura Sostenibilidad, Cultura y Educación.

b) Ayudas a proyectos sobre acciones promotoras de sostenibilidad en la UV. Esta convocatoria tuvo dos ediciones: 2014 y 2015.

c) Cursos de formación. Se realizó un curso de formación continua para PAS: La sostenibilidad en el Campus: ¿Qué podemos hacer?. Curso realizado en 2013, 2014 y 2015. Tres cursos de formación a la demanda para PDI: El aprendizaje/Servicio (ApS): una metodología activa y participativa. Realizado en 2013, 2014 y 2015 en los tres Campus de la UV.

d) Programa de ApS para la promoción de proyectos de Aprendizaje-Servicio. Su objetivo era la institucionalización del ApS en la UV. Desde este programa se impulsó la visualización de proyectos de ApS a través de la grabación de movies sobre proyectos en el curso 2014-2015, así como la constitución de un grupo del profesorado para promover el ApS como proyecto institucional dirigido a poner en relación la construcción del conocimiento profesional práctico con el compromiso social desde un enfoque integrador de los diferentes campos disciplinares y la apertura a los barrios y sus poblaciones socialmente necesitadas de la colaboración solidaria.

e) Sostenibilización Curricular en los planes de Grado (2010-2013): La finalidad de este estudio se ha dirigido a potenciar el desarrollo de competencias para la sostenibilidad a través de los nuevos títulos de Grado puestos en marcha en la UV en el marco del EEES, a través de un profundo análisis: a) de los contenidos de los planes de estudio aprobados por la ANECA, y su relación con las referencias sobre sostenibilidad contenidas en las directrices generales ministeriales $\mathrm{y}$ en las directrices generales sobre sostenibilización curricular aprobadas por la CRUE; b) de la formación para la sostenibilidad ofertada en las Guías docentes de las diversas 
asignaturas en las distintas titulaciones de Grado, con especial énfasis en las competencias generales y específicas descritas, la metodología aplicada y las herramientas didácticas utilizadas. Para este estudio se ha construido, validado y aplicado un instrumento para evaluar la introducción de la sostenibilidad en los planes de estudios universitarios: el Tesauro de Sostenibilidad. Para poder detectar en los textos de los planes de estudio las competencias que hacen referencia a la sostenibilidad se tomó como referencia, los temas prioritarios explicitados por la UNESCO (2005), en el documento de la Década de la educación para el desarrollo sostenible, clasificados según las perspectivas o variables socio-cultural, económica y ecológica que definen el concepto. En base a las cuales se realizó un diagnóstico exhaustivo de la inclusión de competencias generales y especificas relacionadas con la sostenibilidad. Las conclusiones del estudio han permitido transferir los resultados al tejido formativo de la UV a través del envío de Informes parciales a las Comisiones de los diferentes títulos para la posible revisión en el proceso de Modifica de aquellos títulos que no cumplen con la normativa. El instrumento validado Tesauro de Sostenibilidad, se ha transferido a otras instituciones de enseñanza superior, siendo utilizado por algunas de las Universidades que forman parte del Grupo de Trabajo Sostenibilización curricular de la Comisión de Sostenibilidad de la CRUE.

Este trabajo ha merecido la inclusión del Proyecto como ejemplo de Buenas Prácticas con la universidad y el entorno académico, en el archivo de la web de participación del III Encuentro de Rectores Universia.

Los resultados obtenidos se concretaron en: a) Realización de informes sobre la inclusión de competencias para la sostenibilidad de todos los nuevos planes de estudio, que fueron en total 53 grados implantados en la UV (Aznar Minguet, Ull, Piñero y Martínez Agut, 2013a; 2013b); b) Realización de Informes sobre la inclusión de competencias para la sostenibilidad en las Guías docentes de las asignaturas obligatorias y de las asignaturas optativas de los nuevos planes de estudio y su desarrollo mediante la programación de actividades coherentes (Aznar Minguet, Ull, Piñero y Martínez Agut, 2013c), dando lugar a diversas publicaciones: Aznar Minguet, Ull, Piñero, Martínez-Agut y Mondragón, 2013; Aznar, Ull. Piñero y Martínez, 2014; Ull, Aznar, Martínez-Agut y Piñero, 2013a; 2013b.

f) Auto-evaluación del profesorado en materia de sostenibilidad. El objetivo de este estudio iba dirigido a diagnosticar el nivel de introducción de la sostenibilidad en las actividades docentes y facilitar la autoevaluación profesional del profesorado universitario desde criterios de sostenibilidad. Se ha construido y validado en el año 2014, como instrumento de análisis, un cuestionario autodiagnóstico, fundamentado en el referente conceptual propio de la formación de competencias para la sostenibilidad que incluye aspectos cognitivos (conocimientos), procedimentales (técnicas, métodos) y actitudinales (actitudes, valores). Este cuestionario tiene la virtud de utilizarse como instrumento autodiagnóstico para la autoevaluación del tratamiento de la sostenibilidad por parte del profesorado (Aznar et al., 2013; Aznar, Ull, Martínez y Piñero, 2017; Aznar, Ull, Piñero y Martínez, 2017). Y está a disposición del profesorado para su uso on-line. 


\section{La ruta de la sostenibilidad 2016-2030: La educación e investigación para la sostenibilidad desde la perspectiva de los ODS}

La aprobación en 2015, en la Cumbre de Naciones Unidas celebrada en Nueva York del documento Transformar nuestro mundo: la Agenda 2030 para el Desarrollo Sostenible (ONU, 2015) supone un nuevo marco de referencia a nivel mundial. Esta Agenda establece 17 objetivos interconectados (ODS) que pretenden dar respuesta a una compleja gama de desafíos sociales, económicos y medioambientales, y cuyo logro requerirá de profundas transformaciones en nuestras acciones y comportamientos y en el funcionamiento de las sociedades y las economías durante el período 2016-2030.

Entre las aportaciones más importantes de la Agenda 2030 cabe citar: a) La sostenibilidad como elemento central de la propuesta; b) Tratamiento multidimensional de la pobreza, incluyendo su relación con la desigualdad; c) Atención relevante a las cuestiones de género y el empoderamiento de las mujeres y niñas; d) Relevancia de los DD. HH., la justicia y la lucha contra todo tipo de discriminación y e) Reconocimiento de la inviabilidad parcial del modelo de producción y consumo.

Pero cabe también subrayar desde consideraciones críticas, algunas de las principales limitaciones: a) Vaguedad de buena parte de las metas asociadas a los 17 objetivos (buenos propósitos, pero ausencia de compromisos específicos); b) Indefinición sobre los indicadores (ausencia de sistemas estadísticos fiables); c) Ausencia de compromisos concretos sobre los medios de implementación; d) el crecimiento sigue siendo el mismo planteamiento de fondo y e) ausencia de propuestas sobre cambios estructurales.

Con todo, los nuevos ODS introducen un nuevo marco para el desarrollo de la educación e investigación para la sostenibilidad. Y es importante conocer su proceso de instauración para construir resistencias y alternativas desde una perspectiva crítica para avanzar en el desarrollo de la educación e investigación en sostenibilidad.

La educación, fundamental para alcanzar todos estos objetivos, cuenta con su propio objetivo específico, el ODS 4, que se ha propuesto "garantizar una educación inclusiva, equitativa y de calidad y promover oportunidades de aprendizaje durante toda la vida para todos". El Marco de Acción de Educación 2030 ofrece orientación para la aplicación de este ambicioso objetivo y sus compromisos. La educación es la prioridad principal de la UNESCO porque es un derecho humano esencial y es la base para consolidar la paz e impulsar el desarrollo sostenible. Y es muy importante contar con herramientas que permitan llevar esto adelante y cabe destacar dos de ellas aparecidas recientemente.

1. La guía Educación para los Objetivos de Desarrollo Sostenible: objetivos de aprendizaje (UNESCO, 2016), es una herramienta esencial. En ella se indican objetivos específicos de aprendizaje para todos los ODS, descritos en los dominios cognitivo, socioemocional y conductual.

El dominio cognitivo comprende el conocimiento y las herramientas de pensamiento necesarias para comprender mejor el ODS y los desafíos implicados en su consecución. El dominio socioemocional incluye las habilidades sociales que facultan a los alumnos para colaborar, negociar y comunicarse con el objeto de promover los 
ODS, así como las habilidades, valores, actitudes e incentivos de autorreflexión que les permiten desarrollarse. El dominio conductual describe las competencias de acción.

Además, para cada ODS se presentan sugerencias de temas y enfoques pedagógicos. Y se detalla cómo implementar el aprendizaje de los ODS a través de la EDS mediante: la integración de la EDS en políticas, estrategias y programas; los planes de estudio y los libros de textos; la formación docente; la enseñanza de EDS en el aula y en otros entornos de aprendizaje y en la evaluación de los resultados de aprendizaje y la calidad de los programas.

2. La guía Cómo empezar con los ODS en las universidades: una guía para las universidades, los centros de educación superior y el sector académico (SDSN Australia/Pacífic, 2017), en la que se destaca el papel clave que las universidades tienen en contribuir a alcanzar los ODS. La guía proporciona información práctica sobre cómo empezar a trabajar a través de ejemplos concretos aprovechando las experiencias de las universidades que ya han comenzado a involucrarse con los ODS en el sector de Australia/Pacífico. En concreto esta guía recomienda hacer un proceso similar al que ya se hizo en la UV con el Proyecto Campus Sostenible UV/2015, pero con el nuevo marco de los ODS.

Un ejemplo de cómo trabajar este nuevo marco lo encontramos en la investigación iniciada con el profesorado del Máster Universitario en Formación del Profesorado de Educación Secundaria, mediante la formación de grupos de trabajo colaborativos, que han analizado las guías docentes en diferentes talleres y seminarios y ha propiciado una reflexión acerca de la necesidad de prestar más atención a la Sostenibilidad a la luz de los ODS en sus guías y en su práctica docente (Aznar et al., 2018).

\section{Conclusiones}

La sostenibilización curricular requiere decisiones políticas que permitan generar los espacios necesarios para la participación de los diversos estamentos universitarios en la definición de las estrategias institucionales y en el fomento de normas que respondan a los objetivos y valores relacionados con la sostenibilidad.

El relato construido nos permite concluir que la trayectoria de la sostenibilidad en la docencia e investigación en la UV en los últimos 20 años, ha tenido importantes avances; pero también algunos retrocesos; que unos y otros han venido precedidos de políticas universitarias más o menos sensibles a la problemática de la sostenibilidad, cuyas consecuencias han marcado, por una parte las iniciativas pioneras transferidas a otras universidades, pero también la falta de operatividad en proyectos tales como: inclusión de una línea de sostenibilidad en la convocatoria de Proyectos de Innovación en la docencia; Inclusión de items relacionados con la sostenibilidad en la evaluación del profesorado; construcción de un manual de buenas prácticas dirigido a la comunidad universitaria; Creación de materiales didácticos para el desarrollo de competencias para la sostenibilidad, como material de soporte a la docencia; Institucionalización del ApS; Elaboración de un código de ética para la sostenibilidad práctico y transferible a toda la comunidad universitaria, entre otros.

Cuando se inició esta andadura en la UV únicamente los grupos de la red ACES liderados por Geli y Pujol habían iniciado trabajos de investigación sobre la ambientalización curricular, en la Universitat de Girona y en la Autónoma de 
Barcelona; actualmente, el grupo de trabajo de Sostenibilidad en la docencia (CRUE/Sostenibilidad) acoge 17 universidades españolas, en las que hay grupos de investigación ya consolidados o que inician este camino, y el plenario de la CRUE, aprobó en 2012 la revisión de las Directrices sobre la introducción de la sostenibilidad en la docencia universitaria de 2005 que marcan claramente el camino (CRUE Sostenibilidad, 2012). Ahora lo que es necesario es que se implementen dichas directrices en las universidades españolas. Y la aprobación de los ODS 2030 es una nueva palanca para seguir adelante en el camino emprendido hace más de 20 años., porque concreta en los 17 ODS formas de trabajar todos los aspectos de la sostenibilidad que permiten superar algunos de los obstáculos para el avance hacia un futuro sostenible.

\section{Referencias bibliográficas}

Aznar Minguet, P. (2006). El reto educativo de la sostenibilidad en el marco europeo de la educación superior, en A. Escolano Benito, Educación superior y desarrollo sostenible. Madrid: Biblioteca Nueva.

Aznar Minguet, P y Ull, M. A. (2009). La formación de competencias básicas para el desarrollo sostenible: el papel de la Universidad. Revista de Educación, № extraordinario 2009, 219-237. (Ministerio de Educación).

Aznar Minguet, P.; Ull, M.A.; Martínez-Agut, M.P. y Piñero, A. (2017). Evaluar para transformar: evaluación de la docencia universitaria desde el prisma de la sostenibilidad. Revista Enseñanza de las Ciencias, 35(1), 5-27.

Aznar Minguet, P., Ull, M. A., Piñero, A. y Martínez Agut, M. P. (2013a). Informe de investigación: Evaluación de la introducción de Competencias Generales para la Sostenibilidad en los Estudios de Grado de la Universitat de València. http://angelsull.es/sostenibilidad/wpcontent/uploads/2013/04/INFORME_C OMPETENCIAS_GENERALES_GRADOS_CON_PROTECCION_DATOS1.pdf

Aznar Minguet, P., Ull, M. A., Piñero, A. y Martínez Agut, M. P. (2013b). Informe de investigación: Evaluación de la introducción de Competencias Específicas para la Sostenibilidad en los Estudios de Grado de la Universitat de València. http://angelsull.es/sostenibilidad/wpcontent/uploads/2013/04/INFORME_C OMPETENCIAS_ESPECIFICAS_GRADOS_CON_PROTECCION_DATOS1.pdf

Aznar Minguet, P., Ull, M. A., Piñero, A. y Martínez Agut, M. P. (2013c) Informe de investigación: Evaluación de las competencias para la sostenibilidad en las guías docentes de las asignaturas de los títulos de grado de la Universitat de València. https://www.uv.es/fatwireed/userfiles/file/INFORME_GUIAS_DOCENTES_CR EATIVE_COMMONS.pdf

Aznar Minguet, P.; Ull, M. A.; Piñero, A.; Martínez-Agut, M. P. (2014a). La sostenibilidad en la formación universitaria: desafíos y oportunidades. Educación XX1, 17(1), 133-158. (Editada por la UNED).

Aznar Minguet, P.; Ull, M. A.; Piñero, A y Martínez-Agut, M. P. (2014b). Competencias Básicas para la sostenibilidad: un análisis desde el diálogo disciplinar. Revista Bordón. Revista de la Sociedad Española de Pedagogía, 66(2), 13-28. 
Aznar Minguet, P., Ull, M. A., Piñero, A. y Martínez-Agut, M. P. (2014c). El Tesauro de Sostenibilidad. Universidad de Valencia. www.uv.es/acuveg (Sostenibilidad y Educación Superior).

Aznar Minguet, P., Ull, M. A., Piñero, A. y Martínez Agut, M. P. (2015). Competencies for sustainability in the curricula of all new degrees from the University of Valencia (Spain). In M. Barth, G. Michelsen, M. Rieckman and I. Thomas (Eds.), Handbook of higher education for sustainable development. Ch. 32. London: Routledge Publishers.

Aznar Minguet, P., Ull, M. A., Piñero, A. y Martínez Agut, M. P. (2017) La evaluación de la formación de formadores. Un catalizador en el proceso de cambio curricular hacia la sostenibilidad. Revista Iberoamericana de Educación, 73, 225-252.

Aznar Minguet, P., Martínez-Agut, M., Palacios, B., Piñero, A. y Ull, M. A. (2011). Introducing sustainability into university curricula: an indicator and baseline survey of the views of university teachers at the University of Valencia. Environmental Education Research, 17(2), 145-166.

Aznar Minguet, P., Ull, M. A., Piñero, A., Martínez-Agut, M. P. y Mondragón, A (2013) Autodiagnóstico de la Inclusión de la Sostenibilidad en las Actividades Docentes del Profesorado Universitario. https://www.uv.es/evomobile/Recursos/Autodiagnostico_Profesorado.pdf

Aznar Miguet, P., Calero, M., Martínez-Agut, M. P., Mayoral, O, Ull, M. A, Vázquez, V y Vilches, A. (2018). Training Secondary Education teachers through the Prism of Sustainability: The case of the Universitat de València. Sustainability, 10(11), 4170. https://doi.org/10.3390/su10114170

Barrón, A., Navarrete, A. y Ferrer-Balas, D. (2010). Sostenibilización curricular en las universidades españolas. ¿ha llegado la hora de actuar? Revista Eureka Enseñanza y Divulgación de las Ciencias, 7, № extraordinario: La sostenibilidad en la universidad, 388-399.

Barth, M. y Rieckmann, M. (2012). Academic staff development as a catalyst for curriculum change towards education for sustainable development: an output perspective. Journal of Cleaner Production, 26, 28-36.

Barth, M. (2014). Implementing Sustainability in Higher Education. Learning in an age of transformation. London: Routledge Publishers.

Capdevila, I. (1999). L'ambientalització de la universitat. Monografies d'Educació Ambiental № 6. Barcelona: Di7 Edició.

CRUE Sostenibilidad (2012). Directrices para la introducción de la Sostenibilidad en el currículum. CRUE Universidades Españolas. http://www.crue.org/Documentos \%20compartidos/Declaraciones/Directrices_Sosteniblidad_Crue2012.pdf

Campus Sostenible /UV 2015.

https://www.uv.es/cdciencia/pdf/Campus_Sostenible.pdf

(2011).

De Harpe B. y Thomas, I. (2009) Curriculum Change in Universities: Conditions that Facilitate Education for Sustainable Development. Journal of Education for Sustainable Development, 3(1), 75-85. 
Lozano, R., Merrill, M. Y., Sammalisto, K., Ceulemans, K., y Lozano, F. J. (2017). Connecting Competences and Pedagogical Approaches for Sustainable Development in Higher Education: A Literature Review and Framework Proposal. Sustainability, 9(10), 1889. https://doi.org/10.3390/su9101889

Lozano García, F. J., Gándara, G., Perni, O., Manzano, M y Hernández, D. E. (2008). Capacity Building: a course on sustainable development to educate the educators. International Journal in Higher Education, 9(3), 257-281.

Junyent, M. y De Ciurana, A. M. G. (2008). Education for sustainability in university studies: a model for reorienting the curriculum. British Educational Research Journal, 34(6), 762-783.

Junyent, M., Bonil, J. y Calafell, G. (2011). Evaluar la ambientalización curricular de los estudios superiores: un análisis de la red Edusost. Ensino Em Re-Vista, 18(2), 323-340.

Martínez-Agut, M. P., Aznar, P., Ull, A. y Piñero, A. (2007). Promoción de la sostenibilidad en los currícula de la enseñanza superior desde el punto de vista del profesorado: un modelo de formación por competencias. Educatio Siglo XXI, $25,187-208$.

Martínez-Agut, M. P.; Ull, M.A y Aznar Minguet, P. (2014) Education for sustainable development in Early Chilhood Education in Spain: evolution, trends and proposals. European Early Childhood Education Research Journal, 22(2), 213228.

ONU (2015). Objetivos de Desarrollo Sostenible. https://www.un.org/sustainabledevelopment/es/objetivos-de-desarrollosostenible/

Palacios, B. y Aznar Minguet, P. (2004). Ambientalización curricular en los estudios superiores: el caso de la titulación de Pedagogía en la UVEG. En AA.VV. Investigaciones en educación ambiental: de la conservación de la biodiversidad a la participación social. Segovia: Ediciones del Ministerio de Medio Ambiente.

Piñero, A.; Ull, M. A.; Aznar, P. y Martínez, M. P. (2006). Institutional Curriculum Greening for higher education: Towards University Agendas 21, En Educational Paths towards sustainability. Proceedings of 3rd World Environmental Education Congress (3rd WEEC). Torinno (Italy), pp. 248-260, Vol. 1. ISBN: 8885313-17-5.

Piñero, A.; Ull, M. A.; Martínez Agut, M. P. y Aznar, P. (2007). Introducción de la ambientalización en los currícula de las Ciencias Farmacéuticas. Edusfarm, Revista d'educació superior en Farmacia 1, (Facultat de Farmàcia de la Universitat de Barcelona, GDP -Grup de Dinamització Pedagògica-). ISSN: 1886-6271.

SDSN Australia/Pacífico (2017). Cómo empezar con los ODS en las universidades: una guía para las universidades, los centros de educación superior y el sector académico. Edición en español de Red Española para el Desarrollo Sostenible (REDS / SDSN-Spain). 
Sibbel, A. (2009). Pathways towards sustainability through higher education. International Journal of Sustainability in Higher Education, 10(1), 68-82.

Sterling, S. (2005). Higher education, sustainability, and the role of systemic learning. in P. B. Blaze Corcoran and A. E. J. Wals (Eds.), Higher Education and the Challenge of Sustainability: Problematics, Promise and Practice, Ch. 5. Dordretch: Kluwer Academic Press.

Tilbury, D. (2012a). Another World is Desirable: Transforming Higher Education for Sustainability', In S. Sterling, L. Maxey, and H. Luna (Eds.), The Sustainable University: Process and Prospects, London: Earthscan/Routledge.

Tilbury, D. (2012b). Learning to Connect: Personal Reflections along a journey of Education and Learning for a Sustainable Future in the Context of Rio Plus 20. Journal of Education for Sustainable Development, 6(1), 59-62, March 2012.

Thomas, I. (2009). Critical thinking, Transformative Learning, Sustainable Education and Problem-Based learning in Universities. Journal of Transformative Education 7, 245-264.

Ull. M.A. (2014). Competencias para la Sostenibilidad y Competencias en Educación para la Sostenibilidad. Uni-pluri/versidad, 14(3). Artículo de revisión. Revista Universidad Antioquia. Colombia.

Ull, M. A., Aznar, P., Martínez Agut, M. P., Palacios, B. y Piñero, A. (2009). Competencias para la sostenibilidad y currícula universitarios. Revista Enseñanza de las Ciencias, № Extra VIII Congreso Internacional sobre Investigación en Didáctica de las Ciencias, Barcelona, 2964-2967. ISSN: 0212-4521.

Ull, M. A, Aznar Minguet, P., Martínez Agut, M. P., Palacios, B. y Piñero, A. (2010). Conocimientos y actitudes del profesorado universitario sobre problemas ambientales. Revista Enseñanza de las Ciencias 28(3), 433-446.

Ull, M. A., Aznar Minguet, P., Martínez-Agut, M. P. y Piñero, A (2013a). Competencias para la sostenibilidad en los Planes de Estudio de los Grados de Ciencias de la Universidad de Valencia, Revista Enseñanza de las Ciencias, № Extra. Año 2013, Actas IX Congreso Internacional sobre Investigación en Didáctica de las Ciencias, 3406-3411. ISSN: 0212-4521.

Ull, M. A., Aznar Minguet, P., Martínez-Agut, M. P. y Piñero, A. (2013b), Competencias para la sostenibilidad en los planes de estudio de los Grados de Ciencias Sociales y Jurídicas de la Universitat de València, En M. T. Tortosa, J. D. Álvarez, y N. Pellín (eds.), XI Jornadas de Redes de Investigación en Docencia Universitaria: Retos de futuro en la Enseñanza Superior: docencia en investigación para alcanzar la excelencia académica, Alicante: Universidad de Alicante, p. 2432-2445. ISBN 978-84-695-8104-9. http://hdl.handle.net/10045/31305

Ull, M. A.; Martínez Agut, M. P.; Piñero, A y Aznar Minguet, P. (2010). Análisis de la introducción de la sostenibilidad en la enseñanza superior en Europa: compromisos institucionales y propuestas curriculares. Revista Eureka de Enseñanza y Divulgación de las Ciencias, 7, № extraordinario 2010, 413-432. 
Ull, M. A., Martínez-Agut, M. P., Piñero, A. y Aznar Minguet, P. (2014). Perceptions and attitudes of students of teacher-training towards environment and sustainability. Procedia-Social and Behavioral Sciences, 131, 453-457. (Estados Unidos de América: Elsevier, 2014).

Ull, M. A., Piñero, A., Martínez Agut, M. P. y Aznar Minguet, P. (2014). Preconcepciones y actitudes del profesorado de Magisterio ante la incorporación en su docencia de competencias para la sostenibilidad. Enseñanza de las Ciencias, 32(2), 91112.

UNECE (2013). Empowering educators for a sustainable future: tools for policy and practice workshops on education for sustainable development competences. Economic and Social Council. Géneva, 21 and 22 March. GE.13-20120.

UNESCO (2005). Decenio de las Naciones Unidas de la Educación para el Desarrollo Sostenible 2005-14: Plan de aplicación internacional. Proyecto. Paris: UNESCO.

UNESCO (2016). Educación para los Objetivos de Desarrollo Sostenible. Objetivos de Aprendizaje. Educación 2030. Paris, Francia: Ediciones UNESCO.

Vilches, A y Gil, D. (2012). La Educación para la Sostenibilidad en la universidad: el reto de la formación del profesorado. Profesorado, Revista de currículum y formación del profesorado, 16(2), 25-43.

Wiek, A., Withycombe, L. y Redman, Ch. L. (2011). Key competencies in Sustainability: a reference framework for academic program develment. Sustain Sci, 6, 203218. 